\title{
Beneficial effect of a high number of copies of salivary amylase $A M Y 1$ gene on obesity risk in Mexican children
}

\author{
María A. Mejía-Benítez • Amélie Bonnefond • Loïc Yengo • Marlène Huyvaert • \\ Aurélie Dechaume • Jesús Peralta-Romero • Miguel Klünder-Klünder • Jaime García Mena • \\ Julia S. El-Sayed Moustafa • Mario Falchi • Miguel Cruz • Philippe Froguel
}

Received: 14 August 2014 / Accepted: 29 September 2014 / Published online: 14 November 2014

(C) Springer-Verlag Berlin Heidelberg 2014

\begin{abstract}
Aims/hypothesis Childhood obesity is a major public health problem in Mexico, affecting one in every three children. Genome-wide association studies identified genetic variants associated with childhood obesity, but a large missing heritability remains to be elucidated. We have recently shown a strong association between a highly polymorphic copy number variant encompassing the salivary amylase gene ( $A M Y 1$ also known as $A M Y 1 A$ ) and obesity in European and Asian adults. In the present study, we aimed to evaluate the association between $A M Y 1$ copy number and obesity in Mexican children.

Methods We evaluated the number of AMY1 copies in 597 Mexican children (293 obese children and 304 normal weight controls) through highly sensitive digital PCR. The effect of AMY1 copy number on obesity status was assessed using a logistic regression model adjusted for age and sex.
\end{abstract}

María A. Mejía-Benítez and Amélie Bonnefond contributed equally to this study.

\footnotetext{
M. A. Mejía-Benítez · J. García Mena

Departamento de Genética y Biología Molecular, Centro de Investigación y de Estudios Avanzados del Instituto Politécnico

Nacional, Mexico City, Mexico
}

M. A. Mejía-Benítez • A. Bonnefond $\cdot$ L. Yengo $\cdot$ M. Huyvaert $\cdot$

A. Dechaume $\cdot$ P. Froguel

European Genomic Institute for Diabetes (EGID),

FR 3508 Lille, France

M. A. Mejía-Benítez $\cdot$ A. Bonnefond $(\bowtie) \cdot L$. Yengo •

M. Huyvaert $\cdot$ A. Dechaume $\cdot$ P. Froguel $(\bowtie)$

Centre National de la Recherche Scientifique (CNRS) UMR 8199

Lille Pasteur Institute, 1 rue du Prof Calmette,

59019 Lille Cedex, France

e-mail: amelie@good.ibl.fr

e-mail: froguel@good.ibl.fr
Results We identified a marked effect of $A M Y 1$ copy number on reduced risk of obesity (OR per estimated copy 0.84 , with the number of copies ranging from one to 16 in this population; $p=4.25 \times 10^{-6}$ ). The global association between $A M Y 1$ copy number and reduced risk of obesity seemed to be mostly driven by the contribution of the highest $A M Y 1$ copy number. Strikingly, all children with $>10 A M Y 1$ copies were normal weight controls.

Conclusions/interpretation Salivary amylase initiates the digestion of dietary starch, which is highly consumed in Mexico. Our current study suggests putative benefits of high number of $A M Y 1$ copies (and related production of salivary amylase) on energy metabolism in Mexican children.

Keywords $A M Y 1 \cdot A M Y 1 A \cdot$ Childhood obesity · Copy number variation $\cdot \mathrm{CNV} \cdot$ Digital PCR $\cdot$ Genetics $\cdot$ Mexican children $\cdot$ Salivary amylase

M. A. Mejía-Benítez $\cdot$ A. Bonnefond $\cdot$ L. Yengo $\cdot$ M. Huyvaert

A. Dechaume $\cdot$ P. Froguel

Lille 2 University, Lille, France

J. Peralta-Romero $\cdot$ M. Cruz

Medical Research Unit in Biochemistry, Hospital de Especialidades, Centro Médico Nacional Siglo XXI del IMSS, Mexico City, Mexico

M. Klünder-Klünder

Community Health Research Department, Hospital Infantil de México Federico Gómez, Ministry of Health (SSA),

Mexico City, Mexico

J. S. El-Sayed Moustafa $\cdot$ M. Falchi $\cdot$ P. Froguel Department of Genomics of Common Disease, School of Public Health, Imperial College London, Hammersmith Hospital, London, UK 


\section{Abbreviations \\ CNV Copy number variation \\ GWAS Genome-wide association study \\ NCTs Non-template controls \\ SNP Single nucleotide polymorphism}

\section{Introduction}

Childhood obesity has become a major public health problem both in westernised and more recently in developing countries [1]. In Mexico, the prevalence of overweight or obesity among children aged between 5 and 11 years has dramatically increased from $26.9 \%$ in 2006 to $34.4 \%$ in 2012 [2].

Although the epidemic of obesity is attributed mainly to changes in the environment, variations in susceptibility have been attributed to differences in the genetic backgrounds of individuals exposed to similar so-called 'obesogenic' environmental pressures [3]. Indeed, the heritability of BMI has been estimated to range between 40 and $70 \%$ [3, 4]. Moreover, at least one in every 20 obese children carries a single mutation (or chromosome abnormality) causing severe impairment in appetite regulation and early-onset overweight [3].

Nevertheless, the vast majority of obese children are thought to have a polygenic form of obesity. Recently, genome-wide association studies (GWAS) have identified $>60$ frequent single nucleotide polymorphisms (SNPs) that slightly increase BMI [5], and some also increase the risk of early-onset obesity [6-8]. However, $<3 \%$ of the phenotypic variance in BMI has been explained by these loci [5], suggesting that other forms of DNA variation (including copy number variation $[\mathrm{CNV}]$ ) may explain this large missing heritability. In this regard, we recently developed a system biology approach for the identification of potential genedosage effects, combining transcriptomics and GWAS analyses in sibling-pairs discordant for obesity [9]. This approach enabled the identification of a region mapping of both salivary and pancreatic amylase genes ( $A M Y 1$ and $A M Y 2$ [also known as $A M Y 1 A$ and $A M Y 2 B]$, respectively), which was strongly associated with obesity risk and fat mass [9].

Amylase is responsible for starch hydrolysis, and CNV at this locus (ranging from one to 20 copies for $A M Y 1$ ) evolved as an adaptation to dietary habits; populations with high starch consumption carry larger number of copies than others that have maintained an ancestral pre-agriculture way of life [10, 11]. We reported that reduced $A M Y 1$ copy number was associated with increased BMI (change in BMI per copy $=0.15 \mathrm{~kg} / \mathrm{m}^{2}$ ) and obesity risk (OR per copy 1.19) in European and Asian adult populations [9]. Furthermore, AMY1 copy number was shown to be strongly correlated with the amount of serum amylase $[9,10]$. These results provided a presumed genetic link between carbohydrate metabolism and obesity. However, the contribution of $A M Y 1$ copy number to the risk of childhood obesity remains unknown.

In the present study, we assessed the association between AMY1 copy number and obesity risk in 597 Mexican children.

\section{Methods}

Study participants We analysed 597 Mexican children (293 obese children and 304 normal weight controls) from a cohort population fully described elsewhere [12]. The cases and controls were optimally matched for age and sex (Table 1).

BMI-for-age was calculated and classified according to the 2000 Centers for Disease Control and Prevention growth charts [13]. Normal weight was defined as a BMI-for-age below the 85 th percentile, while obesity was defined as a BMI-for-age above the 95th percentile [13]. No information on the pubertal status of the children was available.

Child assent was obtained and parents (or legal guardian) provided written informed consent. The study was approved by the local ethics committees of the Mexican National Health Service ('Instituto Mexicano del Seguro Social', reference number 2011-000-079) and was conducted in compliance with the Declaration of Helsinki.

Digital PCR Digital PCR analysis was performed on the BioMark System (Fluidigm, South San Francisco, CA, USA) using the qdPCR 37K Digital Array (Fluidigm) according to manufacturer's recommendations. This digital array consists of 48 panels, each containing 770 individual reaction chambers. The final reaction mix for a digital panel comprised $7.2 \mathrm{ng}$ DNA (DNA concentration was measured using the Qubit dsDNA HS Assay kit [Life Technologies, Carlsbad, CA, USA]), $2 \mu$ l TaqMan Gene Expression Master Mix (Life Technologies), $0.4 \mu \mathrm{l} 20 \times$ GE Sample Loading Reagent (Fluidigm), $0.2 \mu \mathrm{l}$ AMY1 TaqMan assay (Hs07226362_cn; Life Technologies) and $0.2 \mu \mathrm{l}$ RNase $P$ human TaqMan Copy Number Reference (Life Technologies). A total of $10 \mu$ of $1 \times$ GE Sample Loading Reagent (Fluidigm) with $4 \mu$ sample mix were aliquoted separately into inlet on the digital array and were distributed throughout the partitions within each panel using an automated IFC controller-MX (Fluidigm). Each DNA sample was loaded in quadruplicate, with single

Table 1 Clinical characteristics of the study participants

\begin{tabular}{llll}
\hline Characteristics & Obese cases & Normal weight controls & $p$ \\
\hline$n$ (male/female) & $293(165 / 128)$ & $304(165 / 139)$ & 0.62 \\
Age (years) & $9.5 \pm 1.7$ & $9.5 \pm 1.8$ & 0.88 \\
Height $(\mathrm{cm})$ & $140.0 \pm 11.6$ & $133.3 \pm 11.5$ & $<0.001$ \\
Weight $(\mathrm{kg})$ & $50.2 \pm 12.9$ & $29.9 \pm 7.6$ & $<0.001$ \\
\hline
\end{tabular}

Data are mean \pm SD 
replicate outliers removed. When the estimated copy number of $A M Y 1$ was above 10, the amount of DNA was reduced to $5.4 \mathrm{ng}$ (in the final reaction mix) so as to avoid panel saturation. These DNA samples (with $A M Y 1$ copy number $\geq 10$ ) were finally re-loaded in octuplicate, with single replicate outliers removed. When we observed more than two outliers within the quadruplicate or octuplicate, the digital PCR for those samples was performed again. Outliers were defined as follows: (1) difference in estimated copies of $A M Y 1 \geq 2$; (2) detection of $>600$ positive chambers for $A M Y 1$; and/or (3) detection of $<50$ positive chambers for RNase $P$.

Of note, 11 non-template controls (NTCs) were analysed (using reaction mix with no template DNA). We did not detect any positive chambers in these NTCs.

We and others previously demonstrated that microfluidic digital PCR was a sensitive method to measure highly polymorphic CNVs $[9,14]$.

Statistical analyses The median of AMY1 copy number distribution amounted to six copies. In order to have $50 \%$ of individuals (cases and controls) in the lower half and $50 \%$ of individuals (cases and controls) in the upper half, we randomly ranked individuals with six copies and assigned some of them to the lower half and the others to the upper half.

The effect of AMY1 copy number on obesity status was assessed using logistic regression adjusted for age and sex, under an additive model. We have not adjusted for population stratification. Mexicans are a mixture of Native American, European and African ancestry. Thus, further work would be needed to confirm that the association is not partly confounded. However, the adjustment for the different states of residence of cases and controls (i.e. San Luis Potosí, Queretaro, Tijuana, Guanajuato and Mexico city) did not change (even slightly) the present results (data not shown).

The statistical analyses were performed with SPSS (version 14.0, IBM, Armonk, NY, USA) and R (version 3.0, www.r-project.org/).

\section{Results}

In the 597 Mexican children investigated (including 304 normal weight controls and 293 obese cases), the estimated number of copies of $A M Y 1$ ranged from one to 16 (Fig. 1).

Using a logistic regression model adjusted for age and sex, we found a strong association between AMY1 copy number and reduced risk of obesity (OR per estimated copy 0.84 [95\% CI $0.78,0.91$ ] having in mind that the number of copies ranges from one to 16 in this population; $p=4.25 \times 10^{-6}$ [Fig. 1; Table 2]). The mean number of copies of $A M Y 1$ was $6.1 \pm$ 1.9 in cases compared with $7.0 \pm 2.7$ in controls (Table 2).

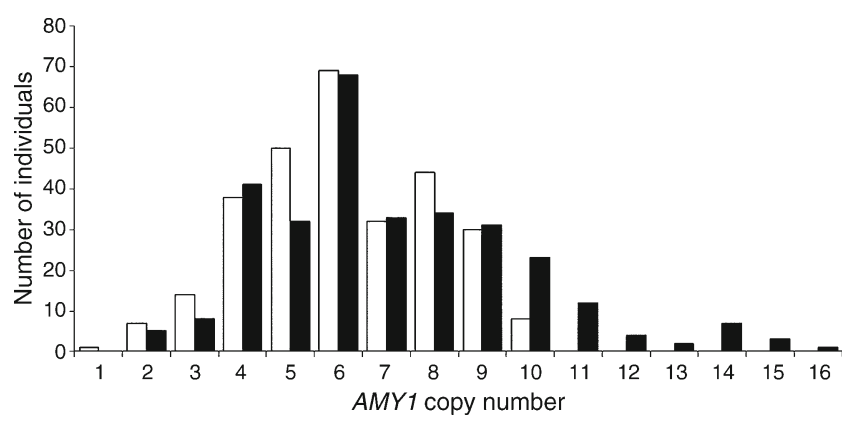

Fig. 1 Distribution of $A M Y 1$ copy number in 597 Mexican children, including 304 normal weight controls (black bars) and 293 obese cases (white bars)

Subsequently, we wondered whether or not the significant effect of $A M Y 1$ copy number on obesity was homogeneous throughout the distribution of $A M Y 1$ copy number within the study group. The mean number of copies of $A M Y 1$ was lower in obese cases than in normal weight controls in the lower or upper half of $A M Y 1$ copy number distribution $(4.6 \pm 1.1$ vs $5.0 \pm 1.1,7.6 \pm 1.2$ vs $9.1 \pm 2.1$, respectively [Table 2]). However, using a logistic regression model adjusted for age and sex, we found that the contribution of $A M Y 1$ copy number to obesity was much stronger in the upper half of $A M Y 1$ copy number distribution than in the lower half (OR per estimated copy $0.54[95 \%$ CI $0.44,0.65] ; p=3.91 \times 10^{-10}$ vs OR per estimated copy 0.76 [95\% CI $0.62,0.94] ; p=0.0122$, respectively [Table 2]). Furthermore, all participants with $>10$ copies of AMY1 ( $n=29$ [Fig. 1]) were normal weight controls. These results suggest that the global association between $A M Y 1$ copy number and reduced risk of obesity might be mostly driven by the contribution of the highest $A M Y 1$ copy number in these Mexican children.

\section{Discussion}

In the present study, we confirmed the marked contribution of AMY1 copy number to reduced obesity risk (OR per estimated copy 0.84$)$. Of note, this effect was similar between the Mexican children included in the current study and our previous meta-analysis that included 2,929 adults of European or Asian origin (593 cases and 2,336 controls) [9]. However, in the present study, we found that the global effect of $A M Y 1$ copy number on reduced obesity risk may be largely driven by the marked contribution of the highest number of $A M Y 1$ copies to obesity risk. While we did not find a significant effect of lower $A M Y 1$ copy number on childhood obesity risk, the highest $A M Y 1$ copy number seems to strongly protect against obesity in Mexican children.

Salivary amylase is a monomeric calcium-binding enzyme produced by the salivary glands that catalyses the initial digestion of dietary starch, which represents $50 \%$ of the total 
Table 2 Effect of $A M Y 1$ copy number on obesity risk in the total number of cases and controls, and in the individuals with $A M Y 1$ copy number above or below the median of $A M Y 1$ copy number distribution (which amounted to six copies)

\begin{tabular}{|c|c|c|c|c|c|c|}
\hline \multirow[t]{2}{*}{ Distribution of $A M Y 1 \mathrm{CN}$} & \multicolumn{2}{|c|}{ Cases } & \multicolumn{2}{|c|}{ Controls } & \multirow[t]{2}{*}{$\mathrm{OR}_{\text {per copy }}(95 \% \mathrm{CI})^{\mathrm{a}}$} & \multirow[t]{2}{*}{$p$} \\
\hline & $n$ & $A M Y 1 \mathrm{CN}$ & $n$ & $A M Y 1 \mathrm{CN}$ & & \\
\hline $\mathrm{CN} \leq 6$ & 146 & $4.6 \pm 1.1$ & 152 & $5.0 \pm 1.1$ & $0.76(0.62,0.94)$ & 0.0122 \\
\hline $\mathrm{CN} \geq 6$ & 147 & $7.6 \pm 1.2$ & 152 & $9.1 \pm 2.1$ & $0.54(0.44,0.65)$ & $3.91 \times 10^{-10}$ \\
\hline Total & 293 & $6.1 \pm 1.9$ & 304 & $7.0 \pm 2.7$ & $0.84(0.78,0.91)$ & $4.25 \times 10^{-6}$ \\
\hline
\end{tabular}

Data are means $\pm \mathrm{SD}$

${ }^{a}$ OR per $A M Y 1$ copy from a logistic regression adjusted for age and sex, under an additive model

$\mathrm{CN}$, copy number

carbohydrate content of the US diet [15]. Importantly, Perry et al demonstrated that populations with starchy food resources carried, on average, more AMY1 copies than those with traditional diets with few starchy foods $(6.7 \pm 2.3$ vs $5.4 \pm$ 2.0 , respectively) [10]. The Mexican population has a diet rich in various starchy foods (comprising maize, wheat and different varieties of common bean) [16]. In Mexico, the daily consumption of corn tortilla has been estimated at approximately 325 g, supplying $70 \%$ of the calories and half of the proteins, and the available starch content in the tortilla has been estimated to be $63-73 \%$ following processing conditions (compared with $80 \%$ for white bread) [17]. It was proposed that higher amount of salivary amylase (which correlate with greater numbers of $A M Y 1$ copies) confers a fitness advantage for individuals consuming a highly starchy diet by improving digestion in the oral cavity, stomach and intestines [10].

A growing body of research from the pharmaceutical industry has focused on products that slow the absorption of carbohydrates (which is the greatest source of calories in most diets) in an effort to improve glycaemic control and reduce weight [18]. In particular, a recent clinical study showed that the use of an inhibitor of salivary amylase (extracted from common white bean) was associated with modest decrease in weight (on average, $2.9 \mathrm{~kg}$ in 12 weeks) [19], which seems to be in contradiction with the findings of our study. However, it is noteworthy that the expression of $A M Y 1$ is not restricted to salivary glands. We previously showed a high $A M Y 1$ expression in adipose tissue [9]. Therefore, salivary amylase may also act beyond nutrient digestion in the mouth. In this respect, a recent mouse genetic linkage study showed that a SNP (rs29982345) close to Amyl was significantly associated with weight gain in $>100$ different strains of mice fed a high-fat, high-sucrose diet [20]. The same SNP showed significant association with an enrichment of the genera Enterobacteriaceae in the gut microbiota of the mice [20], which was reported to be correlated with obesity in humans [21]. Therefore, salivary amylase may contribute to modulation of the gastrointestinal tract function in a way that remains to be fully explored.
Recently, a study has demonstrated that non-obese adults with high salivary amylase activity (and high AMY1 copies) presented with improved glycaemic control following liquid starch ingestion (and not after oral glucose load) [22]. The findings from that study along with our results suggest the putative benefits of carrying a higher number of $A M Y 1$ copies and of the highly related production of salivary amylase on glucose and energy metabolism, in particular in populations like Mexicans with a diet rich in starch.

Acknowledgements We are sincerely indebted to all participants of the present study.

Duality of interest The authors declare that there is no duality of interest associated with this manuscript.

Contribution statement MAMB contributed to acquisition of data and to writing the manuscript. $\mathrm{AB}$ designed the study, performed the statistical analyses and wrote the manuscript. LY performed the statistical analyses and contributed to the discussion. $\mathrm{MH}$ and $\mathrm{AD}$ contributed to acquisition of data and reviewed/edited the manuscript. JGM, JPR and MKK reviewed/edited the manuscript and contributed to acquisition of data. JSESM and MF contributed to interpretation of data and reviewed/ edited the manuscript. MC contributed to acquisition of data and reviewed/edited the manuscript. PF designed the study, wrote the manuscript and is the guarantor of this work. All the authors approved the final version of the submitted draft.

Funding The study was supported by the Centre National de la Recherche Scientifique (CNRS). MAMB was supported by CONACyT Fellowship \#233834 and by Cinvestav.

\section{References}

1. Waters E, de Silva-Sanigorski A, Hall BJ et al (2011) Interventions for preventing obesity in children. Cochrane Database Syst Rev, Issue 12, Art no.:CD001871

2. Encuesta Nacional de Salud y Nutrición (2012) Resultados Nacionales. Inst Nac Salud Pública http://ensanut.insp.mx/ informes/ENSANUT2012ResultadosNacionales.pdf

3. El-Sayed Moustafa JS, Froguel P (2013) From obesity genetics to the future of personalized obesity therapy. Nat Rev Endocrinol 9:402413 
4. Hemani G, Yang J, Vinkhuyzen A et al (2013) Inference of the genetic architecture underlying BMI and height with the use of 20 , 240 sibling pairs. Am J Hum Genet 93:865-875

5. Berndt SI, Gustafsson S, Mägi R et al (2013) Genome-wide metaanalysis identifies 11 new loci for anthropometric traits and provides insights into genetic architecture. Nat Genet 45:501-512

6. Meyre D, Delplanque J, Chèvre J-C et al (2009) Genome-wide association study for early-onset and morbid adult obesity identifies three new risk loci in European populations. Nat Genet 41:157-159

7. Wheeler E, Huang N, Bochukova EG et al (2013) Genome-wide SNP and CNV analysis identifies common and low-frequency variants associated with severe early-onset obesity. Nat Genet 45:513-517

8. Bradfield JP, Taal HR, Timpson NJ et al (2012) A genome-wide association meta-analysis identifies new childhood obesity loci. Nat Genet 44:526-531

9. Falchi M, El-Sayed Moustafa JS, Takousis P et al (2014) Low copy number of the salivary amylase gene predisposes to obesity. Nat Genet 46:492-497

10. Perry GH, Dominy NJ, Claw KG et al (2007) Diet and the evolution of human amylase gene copy number variation. Nat Genet 39:1256-1260

11. Santos JL, Saus E, Smalley SV et al (2012) Copy number polymorphism of the salivary amylase gene: implications in human nutrition research. J Nutrigenet Nutrigenomics 5:117-131

12. Mejía-Benítez A, Klünder-Klünder M, Yengo L et al (2013) Analysis of the contribution of FTO, NPC1, ENPP1, NEGR1, GNPDA2 and MC4R genes to obesity in Mexican children. BMC Med Genet 14:21

13. Kuczmarski RJ, Ogden CL, Guo SS et al (2002) 2000 CDC Growth Charts for the United States: methods and development. Vital Health Stat 11:1-190
14. Whale AS, Huggett JF, Cowen S et al (2012) Comparison of microfluidic digital PCR and conventional quantitative PCR for measuring copy number variation. Nucleic Acids Res 40:e82

15. Mandel AL, Peyrot des Gachons C, Plank KL et al (2010) Individual differences in AMY1 gene copy number, salivary $\alpha$-amylase levels, and the perception of oral starch. PLoS One 5:e13352

16. Sáyago-Ayerdi SG, Tovar J, Zamora-Gasga VM, Bello-Pérez LA (2014) Starch digestibility and predicted glycaemic index (pGI) in starchy foods consumed in Mexico. Starch 66:91-101

17. Tovar J, Sáyago-Ayerdi SG, Peñalver C et al (2003) In vitro starch hydrolysis index and predicted glycemic index of corn tortilla, black beans (Phaseolus vulgaris L.), and Mexican "taco. Cereal Chem 80: 533-535

18. Barrett ML, Udani JK (2011) A proprietary alpha-amylase inhibitor from white bean (Phaseolus vulgaris): a review of clinical studies on weight loss and glycemic control. Nutr J 10:24

19. Grube B, Chong W-F, Chong P-W, Riede L (2014) Weight reduction and maintenance with IQP-PV-101: a 12-week randomized controlled study with a 24-week open label period. Obesity (Silver Spring) 22:645-651

20. Parks BW, Nam E, Org E et al (2013) Genetic control of obesity and gut microbiota composition in response to high-fat, high-sucrose diet in mice. Cell Metabol 17:141-152

21. Karlsson CLJ, Onnerfält J, Xu J et al (2012) The microbiota of the gut in preschool children with normal and excessive body weight. Obesity (Silver Spring) 20:2257-2261

22. Mandel AL, Breslin PAS (2012) High endogenous salivary amylase activity is associated with improved glycemic homeostasis following starch ingestion in adults. J Nutr 142:853-858 